EPiC Series in Computing
Volume 70, 2020, Pages 21-32
$\begin{gathered}\text { Proceedings of the 12th International Conference } \\ \text { on Bioinformatics and Computational Biology }\end{gathered}$

\title{
In silico library design, screening and MD simulation of COX-2 inhibitors for anticancer activity
}

\author{
Ankita Sahu ${ }^{1}$, Dibyabhaba Pradhan ${ }^{2}$, Khalid Raza $^{3 *}$, Sahar Qazi $^{3}$, \\ A K Jain ${ }^{4}$, Saurabh Verma ${ }^{1}$ \\ ${ }^{1}$ Tumor Biology, ICMR-National Institute of Pathology, New Delhi, India-110029 \\ ${ }^{2}$ ICMR-AIIMS, Computational Genomics Centre, Indian Council of Medical Research, India-110029 \\ ${ }^{3}$ Department of Computer Science, Jamia Millia Islamia, New Delhi-110025 \\ ${ }^{4}$ Biomedical Informatics Centre, ICMR-National Institute of Pathology, New Delhi, India-110029 \\ *kraza@jmi.ac.in
}

\begin{abstract}
Health problems are increasing worldwide pertaining to cancer modalities. Cyclooxygenase enzyme is known to be involved in cancer biology, neurological disorders, cardiovascular and other diseases. It has been a promising target for developing novel anti-inflammatory drugs in breast cancer treatment. Hence, a computer-aided drug design strategy was applied to identify potent inhibitors of the COX-2 receptor. For this purpose, 12084 ligands from different databases to be tested based on similarity search criteria and were docked against our target protein COX-2 retrieved from the protein data bank. The high-throughput virtual screening protocol was performed and examined the compounds for its binding free energies. Eleven compounds were found out with better binding affinity by virtual screening results and showed interaction with the protein at the known active site. The selected compounds filtered through the Lipinski's rule of five. The physicochemical properties and bioactivity scores were calculated. Molecular docking calculations, MD simulations, ADMET properties, and protein-ligand interaction were analyzed to determine the suitability of each ligand. Overall, the results from our study suggest that compound ZINC000039428234 could be a potent inhibitor for the COX-2 protein of breast cancer. We look forward to this result is of the enormous key in designing a potential drug candidate for breast cancer.
\end{abstract}

Keywords: COX-2 enzyme, Ramachandran Plot, Molecular docking, Prime MMGBSA, MD simulation, ADMET properties 


\section{Introduction}

Cyclooxygenase (COX)enzyme, generally known as prostaglandin-endoperoxide synthase (PTGS), is responsible for the important biological mediators termed prostanoids, including prostaglandin, thromboxane, and prostacyclin[1,2]. It exists into two isoforms referred to as COX-1 and COX-2. Overexpression of COX-2 associated with various types of cancers, including breast, ovarian, melanoma, colon, glioblastoma, prostate, etc. result in cell mortality[3,4]. We are trying to identify some new lead compounds for breast cancer to better management of inflammatory conditions via targeting the COX-2 enzyme based on the in silico study.

A drug or small molecules may regulate the biological processes in the structure consequence of their size and less complexity. These are well-connected stands on structure and activity. The activity plays an essential role in protein binding and other pharmacological and physiological perspectives; generally, frequently evaluate anti-inflammatory and anti-cancerous activities. Several numbers of chemicals that necessitate evaluation regulate the chemicals that have made in the traditional phase. It is not possible for the science to evaluate every chemical with the most rigorous testing strategies, so computational studies are useful to approach viability, reduce the time, cost, and effectiveness. It is central to note that deep learning requires the biochemical and chemical nature with ligands and receptors. Accurate treatment of each component is essential to compute binding free energies in the multidisciplinary research field that combine the understanding of molecular biology, biochemistry, chemistry, biophysics and, computer sciences. The COX-2 protein is a noticeable impact in new potential drug designing candidates, which deals with several diseases, including breast cancer[3,5,6].

Molecular docking and virtual screening (VS) techniques are utilized to the identification of binding sites and small molecule confirmation as well as to find a potent drug[7,8]. There are numerous reports displayed that the VS method was fruitfully used in making qualitative predictions that showed the difference between active and inactive compounds against a specific target $[9,10]$. The early prediction of ADMET (Absorption, Distribution, Metabolism, Excretion, and Toxicity) properties plays a perceptible impact in enhances the success rate of compounds reaching the drug development stage[11]. Here, the computational approach, such as virtual screening, ADMET and molecular dynamic simulation have been employed, which promotes the effective drug molecule for COX-2 protein.

In our current study, we speculate a computational approach to develop a potent inhibitor that could be helpful in potential lead compounds with better activity for COX-2 targeted therapy.

\section{Materials and methodology}

\subsection{Schrodinger suite and activities of molecular modelling}

Schrodinger suite Maestro 16.4 building window was used to carrying out the molecular docking studies in Dell Optiplex 7050 computer system. Maestro is the Graphical User Interface (GUI) for the in-silico module and containing necessary molecular capabilities such as data building, loading, viewing the structure, modifying and optimize the small molecule for estimating the results. The selected therapeutic target COX-2 belongs to a large family of biomolecules [12]. Licensed software and some other online resources were used for the carrying out of the computational method.

\subsection{Protein Preparation}

In silico study was conducted to assess the potency of the Cyclooxygenase- 2 receptor. The 3D structure of the COX-2 receptor (PDB ID: 5IKR) was obtained from the Research Collaboratory for Structural Bioinformatics Protein Data Bank (www.rcsb.org) [13] represented in Figure 1. It made up of heavy atoms and includes a co- crystallized ligand named2-[(2,3-Dimethylphenyl)amino]benzoic 
acid(ID8), water molecules, metal ions, and cofactors. Hydrogen atoms were added, whereas bond orders and formal charges were assigned for protein preparation. This molecule was optimized at neutral $\mathrm{pH}$ and then the structure was minimized using an optimized potential for liquid simulations (OPLS-2003) force field[14].

\subsection{Structure Validation by Ramachandran Plot}

The constructed structure was validated by the Ramachandran plot using the PROCHECK server[15,16]. It is a simple plot for measuring with tight clustering of phi $\sim-50$ and psi $\sim-50$. The dihedral angles $\varphi$ against $\psi$ of amino acid residues in the selected protein structure can be visualized[17]. It is determined by the percentage of residues in the most favored, allowed, disallowed (bad contents) regions to checked for Ramachandran core values.

\subsection{Ligand library preparation}

Initially, we downloaded different ligands (12084 compounds) in smile format from various databases such as Zinc15 (http://zinc15.docking.org/) [18-20], ChemSpider database (http://www.chemspider.com/) [21,22] and BindingDB (https://www.bindingdb.org/)[23]based on the similarity-based search with Tanimoto coefficient $(\mathrm{Tc}) \geq 0.70$ that share similar activity $(70 \%)$ between two or more descriptors[24]. Ligands were prepared using the Epik2.0 with the $\mathrm{pH}$ range of $7 \pm 2$ andSchrodinger LigPrep module[25,26]. The ligand preparation was instigated, such as torsional (dihedral) is stepped modified and adjusting the chemical correctness (protonation), tautomers, ring conformations, stereoisomer, and eliminate molecules using several conditions including molecular weight or particular types and numbers of functional groups present[18]. Generated 3D ligand structures from ligprep produces a single and low- energy with correct chiralities for each fruitfully processed identical input structure. Further, the OPLS-2005 force field was applied for optimizing the 3D structure of the ligand.

\subsection{Molecular docking}

The grid is a three-dimensional box that represents the area of the receptor to be docked. It also covers the size of the active site arbitrarily and determines the position. Overall, 12084 prepared compounds from different databases were docked at the centroid of the co-crystallized binding site ligand (2-[(2,3-dimethyl phenyl)amino]benzoic acid) for calculating the binding affinity of the ligand interactions with target protein represented in Figure 1(C). The default parameters of van der Waals radius scaling factor 1.00 and charge cut-off of 0.25 around the receptor grid generation (5IKR) subjected to OPLS-2005 power field in glide using Glide v6.3 module(Grid-based Ligand Docking with Energetics) [18-20]. The virtual screening workflow was performed to a detailed analysis of potential small molecule inhibitors against the assumed binding site of COX-2 protein. The procedure constraints algorithm was applied, such as Run QikProp, Lipinski filter, and reactive filter and set to filter. Glide module of Schrodinger Suite HTVS, Standard Precision, and Extra Precision (XP) parameters was applied for good scoring results compared with the native ligand. Finally, we selected the best outcome with lower docking energies for biological testing based on the GlideScore, Glide energy, hydrophobic and hydrogen bonding.

Prime MMGBSA (molecular mechanics- generalized Born surface area) module of Schrodinger was also performed to determine the estimation of binding affinities of the likely ligands binding modes within the docking result using the default parameter settings[27]. The "MMGBSA $\Delta G$ Bind" calculation $[d G(1)]$ was done by following an equation

$$
d G(1)=E \_c o m p l e x(\text { minimized })-\left(E \_l i g a n d(\text { minimized })+E \_r e c e p t o r(\text { minimized })\right)
$$


where MMGBSA denotes molecular mechanics energies combined with the generalized Born and surface area continuum solvation; $d G$ bind denotes the calculated relative free energy of ligand and receptor; $E \_$complex is the MM/GBSA energy of the minimized complex, E_receptor is MM/GBSA energy of protein (unbound, minimized) without ligand, and E_liganddenotes the MM/GBSA energy of the ligand after removing it from the complex.

\subsection{In silico toxicity assessment}

There are $60 \%$ of all drugs in the clinical trial phase, which fails due to their harmful or toxic effect. Thus, computational study plays a significant role in the pharmacokinetic pipeline of potential drug design development[11,28]thereby, it minimizes the development cost and time. The druglikeliness of all the best compounds was calculated through the pharmacokinetic profile and Lipinski's rule of five using the in-silico analysis approach. It gives an idea about drug-like properties that work with five descriptors, including molecular weight (MW), LogP value, Hydrogen acceptor (nON), Hydrogen donor (nOHNH) and Topological Polar Surface Area (TPSA)[29,30]. The best-predicted results were screened for ADMET properties using the QikProp module and Molinspiration open access online software. These properties are essential for a compound to be an active drug.

\subsection{Protein-ligand interaction}

Protein-ligand interaction of docked structure is an important step to perform their biological function. Figure 2 describes the hydrogen-bond and hydrophobic interaction outline between the compounds and the main or chain elements of the protein. Protein-ligand interactions were performed through ligand interaction Maestro 16.4 module based on the best score of docking calculation. These interactions were further studied for checking simulation time after the simulation process.

\subsection{Molecular Dynamics (MD) Simulations}

Molecular dynamics simulations were performed using different software such as - CABS Flex 2.0 for 100 cycles, 100 trajectory frames for $10 \mathrm{~ns}$ with some additional distance restraints with a global weight of 1.0 [35] and Bluues [36], based on Poisson-Boltzmann/Generalized Born (PB/GB) molecular mechanics the solvent probe radius was set as $1.4 \AA$, minimum atomic radius $1 \AA$, salt radius $2 \AA$, ionic strength 0.15 at $298 \mathrm{~K}$., in order to analyze the conformational stability of the receptorligand complex system represented in figure 3. We also executed Vienna-PTM molecular dynamics simulation using force field parameters GROMOS 45A, 54A7, 54A8 respectively to determine any possible post-translational modifications present in the protein complex subjected to simulation [37].

The fluctuation of the individual amino acid residues of the best hit was explained based on the root mean square fluctuation (RMSF) values using - CABS Flex 2.0, in order to analyze the conformational stability within a nanosecond time scale of the receptor-ligand complex system. The highest RMSF value reflects more flexibility, whereas the lowest value implies the limited motion of the system during the simulation process. We also executed Vienna-PTM molecular dynamics simulation to determine any possible post-translational modifications present in the protein complex subjected to simulation [37].

\section{Results and Discussion}

With the aid of the modules as mentioned above, it was possible to carry out molecular docking, lead optimization (2D/3D and relative binding affinity prediction), molecular dynamics simulation and visualization. Ramachandran plot gives information about with and without energy minimized structure of COX-2 structure (PDB ID 5IKR). The minimized structure expresses more accurate and 
flexible than without energy minimized outcome [15] and explains the prepared protein is stereochemically stable, represented in Figure 1(A, B). The distribution of dihedral phi/psi angles of amino acid residues (90.1\%) in the most favoured regions (red), followed by $9.7 \%$ residues, present in the additional allowed regions(yellow), whereas $0.2 \%$ residues were present in the generously allowed region indicated in Figure 1(B). While the white region $(0.0 \%$ residue) represents sterically disallowed regions for all amino acids except glycine due to its lack of the side chain. The prepared protein was used for further docking study.

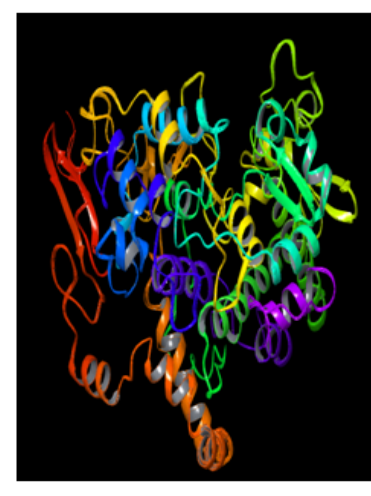

A

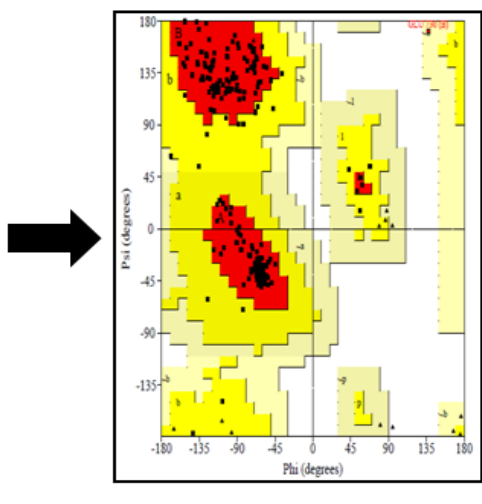

B

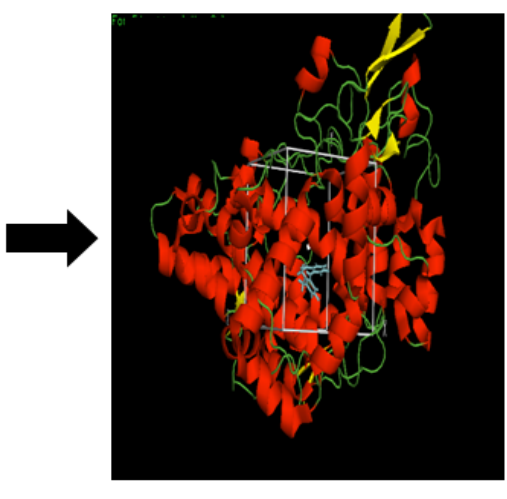

C

Figure 1: (A) Crystal structure of Human Cyclooxygenase (PDB id: 5IKR(B) Ramachandran Plot (C) Grid box at the potential binding site of COX-2 enzyme

Virtual screening of the compounds from three different databases (ZINC database 1207 ligands; ChemSpider 9894 ligands; Binding Database 983 ligands) was performed to finding out the topscoring compounds [31]. We reduced the search space of active compounds using 'similar target search' criterion with the Tanimoto coefficient $(\mathrm{Tc}) \geq 0.70$ to prepare in-house databases of the COX2 receptor. Based on XP score, 11 best compounds have been selected with a higher glideScore ranged between -7.955 to $-10.738 \mathrm{kcal} / \mathrm{mol}$ than that of the native compound indicated in Table 1 . COX-2 inhibitor against COX-2 target suggesting them as potential hits for drug development. Besides, the prime MM-GBSA also calculated for estimating the binding free energy for 11 compounds. Binding energy calculation through MM-GBSA is considered much accurate than the XP Score. It is encouraging to note that, top hit molecule has higher potency to inhibit the COX-2 protein, was used for further study.

Lipinski's rule of five, also referred to as Rule of five (RO5) and Pfizer's rule of five, offers the valuable tool to assess the drug-likeness properties of molecules [29,30,32]. According to this rule, the higher bioactivity score indicates greater the chance of the specific compound will be active. It plays a significant role in drug design and development to the final clinical success of drug candidates. The outcome results were all compounds following the Lipinski's rule of five as well as bioactivity score for finding the more suitable result including GPCR ligand, ion channel modulator, a kinase inhibitor, protease inhibitor and Enzyme inhibitor as shown in Table 1 and Table 2. Overall, ADMET profiles were measured and showed a lower value that indicates the better draggability using Qikprep program and Molinspiration software. Subsequently, the best compounds with fitness scores > 1.0 were observed for their pharmacokinetic profile, such as Lipinski's parameter, QPlogPo/w, QPloghERG, QPPCaco, QPlogBB, and logKhsa. The partition coefficient QPlogPo/w value provides the estimation of absorption and distribution of drugs within the body ranged from1.193 to0.967. The QPPCaco predicted in Caco cells was measured to be good mimic for the gutblood barrier ranged from 367.768 to1823.826. However, the $\operatorname{logBB}$ predicted brain/blood 
permeability acceptable range from -0.942 to -1.162 and logKhsa used to predict the binding to human serum albumin values range-1.086 to -0.680 .

\begin{tabular}{|c|c|c|c|c|c|c|c|c|c|c|c|}
\hline $\begin{array}{l}\text { S. } \\
\text { No }\end{array}$ & Title & $\begin{array}{c}\mathrm{XP} \\
\text { Score }\end{array}$ & MMGBSA & $\begin{array}{l}\text { Mol } \\
\text { MW } \\
<500\end{array}$ & $\begin{array}{c}\text { Donor } \\
\text { HB }<5\end{array}$ & $\begin{array}{c}\text { Accpt } \\
\text { HB 2-10 }\end{array}$ & $\begin{array}{l}\text { PSA } \\
(\stackrel{140}{140} \\
\AA\end{array}$ & $\begin{array}{c}\text { QPlog } \\
\text { Po/w } \\
(-2.0 \text { to } \\
6.5)\end{array}$ & $\begin{array}{c}\text { QPPCaco } \\
(\mathrm{nm} / \mathrm{sec}) \\
<25 \\
\text { poor, }>50 \\
0 \text { great })\end{array}$ & $\begin{array}{c}\text { QPlogBB } \\
(-3 \text { to } \\
1.2)\end{array}$ & $\begin{array}{c}\text { QPlog } \\
\text { Khsa } \\
(-1.5 \text { to1.5) }\end{array}$ \\
\hline 1. & ZINC000039428234 & -10.738 & -67.654 & 299.99 & 0 & 2.0 & 49.76 & 0.967 & 877.455 & -0.767 & -0.687 \\
\hline 2. & ZINC000000244236 & -10.378 & -63.321 & 284.18 & 0 & 7.0 & 94.62 & 0.523 & 367.768 & -1.162 & -1.086 \\
\hline 3. & Compound A & -10.238 & -64.081 & 300.25 & 0 & 6.5 & 64.67 & 0.291 & 630.665 & -0.529 & -1.060 \\
\hline 4. & ZINC000196009905 & -10.211 & -64.968 & 260.22 & 0 & 4.5 & 53.15 & 1.577 & 1823.826 & -0.307 & -0.700 \\
\hline 5. & ZINC000130010902 & -10.160 & -63.957 & 261.63 & 0 & 5.5 & 65.87 & 0.668 & 755.781 & -0.312 & -0.930 \\
\hline 6. & ZINC000039914985 & -10.059 & -66.457 & 256.17 & 0 & 5.0 & 60.47 & 1.457 & 1705.200 & -0.328 & -0.680 \\
\hline 7. & Compound B & -10.028 & -64.042 & 281.34 & 0 & 6.0 & 59.74 & 1.843 & 689.995 & -0.577 & -0.879 \\
\hline 8. & ZINC000048442590 & -10.007 & -62.951 & 224.18 & 0 & 4.5 & 54.16 & 1.215 & 1162.003 & -0.431 & -0.758 \\
\hline 9. & ZINC000000000348 & -9.748 & -68.009 & 272.17 & 0 & 5.5 & 68.19 & 1.375 & 1708.488 & -0.411 & -0.785 \\
\hline 10. & ZINC000000351310 & -9.736 & -63.343 & 244.16 & 0 & 5.0 & 61.95 & 1.193 & 1705.270 & -0.313 & -0.818 \\
\hline 11. & ZINC000039127665 & -7.955 & -63.859 & 249.76 & 0 & 3.0 & 51.24 & 0.785 & 1254.663 & -0.942 & -0.976 \\
\hline 12. & $\begin{array}{c}\text { Reference } \\
\text { Compound (ID8) }\end{array}$ & -9.751 & -68.190 & 271.16 & $\mathbf{0}$ & 7.0 & 53.93 & 0.273 & 1045.872 & -0.266 & -1.391 \\
\hline
\end{tabular}

Table 1. Predicted binding affinity and ADME Properties of compounds using maestro program

These results of the ADMET profile of all selected compounds were found to be within the acceptable range that useful for the human following the drug-likeness properties of the compounds thus suggests

\begin{tabular}{|c|c|c|c|c|c|c|c|c|c|c|c|c|c|c|c|c|}
\hline $\begin{array}{l}\dot{z} \\
\dot{n}\end{array}$ & 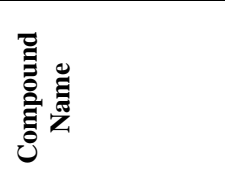 & 串 & 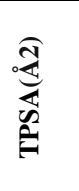 & 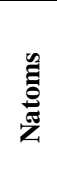 & $\begin{array}{l}\dot{3} \\
\dot{\vec{\theta}}\end{array}$ & Z & 音 & 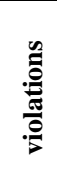 & $\stackrel{\vec{E}}{\vec{\Xi}}$ & $\frac{g}{\mathrm{E}}$ & 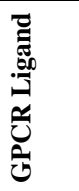 & $\sum_{U}^{\sum}$ & 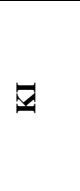 & 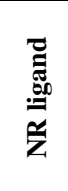 & 总言 & 总苛 \\
\hline 1. & ZINC000039428234 & 5.21 & 29.10 & 20 & 299.68 & 2 & 1 & 1 & 4 & 231.76 & -0.29 & -0.21 & 0.01 & -0.23 & -0.46 & -0.26 \\
\hline 2. & ZINC000000244236 & 2.84 & 69.68 & 22 & 298.29 & 5 & 0 & 0 & 7 & 263.74 & -0.37 & -0.52 & -0.66 & -0.21 & -0.43 & -0.28 \\
\hline 3. & Compound A & 4.70 & 29.10 & 17 & 245.71 & 2 & 1 & 0 & 3 & 216.94 & -0.52 & -0.27 & -0.35 & -0.36 & -0.96 & -0.38 \\
\hline 4. & ZINC000196009905 & 3.35 & 43.38 & 19 & 272.32 & 3 & 0 & 0 & 5 & 237.09 & -0.26 & -0.49 & -0.74 & -0.37 & -0.24 & -0.19 \\
\hline 5. & ZINC000130010902 & 3.17 & 81.14 & 19 & 275.74 & 4 & 5 & 0 & 3 & 239.51 & -0.27 & -0.25 & -0.09 & -0.43 & -0.42 & -0.03 \\
\hline 6. & ZINC000039914985 & 3.79 & 52.61 & 20 & 270.28 & 4 & 0 & 0 & 5 & 244.51 & -0.39 & -0.24 & -0.43 & -0.07 & -0.40 & -0.20 \\
\hline 7. & Compound B & 3.73 & 55.12 & 18 & 260.72 & 3 & 3 & 0 & 3 & 228.22 & -0.35 & -0.31 & -0.08 & -0.48 & -0.54 & -0.21 \\
\hline 8. & ZINC000048442590 & 3.50 & 55.12 & 18 & 240.31 & 3 & 3 & 0 & 3 & 231.25 & -0.31 & -0.31 & 0.05 & -0.50 & -0.38 & -0.13 \\
\hline 9. & ZINC000000000348 & 3.00 & 61.84 & 21 & 286.28 & 5 & 0 & 0 & 6 & 253.50 & -0.33 & -0.21 & -0.37 & -0.14 & -0.35 & -0.17 \\
\hline 10. & ZINC000000351310 & 3.39 & 52.61 & 19 & 256.26 & 4 & 0 & 0 & 5 & 227.95 & -0.40 & -0.17 & -0.49 & -0.14 & -0.40 & -0.17 \\
\hline 11. & ZINC000039127665 & 5.63 & 49.33 & 19 & 296.15 & 3 & 2 & 1 & 3 & 238.49 & -0.24 & -0.17 & -0.21 & -0.10 & -0.61 & -0.17 \\
\hline 12 & $\begin{array}{c}\text { Reference } \\
\text { compound (ID8) }\end{array}$ & 4.77 & 49.33 & 18 & 241.29 & 3 & 2 & 0 & 3 & 227.98 & -0.28 & -0.20 & -0.15 & -0.16 & -0.50 & -0.10 \\
\hline
\end{tabular}

Table 2. Predicted Molecular and bioactivity properties using Molinspiration software

[miLogP: $\log$ P (partition coefficient); TPSA: Topological Polar Surface Area; natoms: number of atoms; nON: hydrogen bond acceptor; nOHNH: hydrogen bond donor; nviolations: number of violations; nrotb: number of rotatable bonds; GPCR: G protein-coupled receptor;ICM:Ion Channel Modulator; KI Kinetic inhibitor; NR ligand : Nuclear receptor ligand] 
their potential as druggable molecules. Hence, the prediction of these properties may shed light on the earlier procedure of reducing the failure rate for further investigation in the future. Among all compounds, higher the binding affinity was observed in compound ZINC000039428234 (-10.738 $\mathrm{kcal} / \mathrm{mol}$ ), which indicates the good-quality results for COX-2 enzyme. This compound was selected for further studies such as protein-ligand interaction and RMSF analysis based on the least binding energy (GlideScore and MMGBSA score) with numerous biological activities.

Hydrogen bond and hydrophobicity interaction for efficient absorption and distribution are essential for the site of action in the body. Hydrogen bond interactions finding between the ligand and target protein residues are encountered at the binding site. Hydrogen bond was observed constituting Tyr385 amino acid residues in the protein-ligand interaction module. Residue Arg 120 located near to the trifluoromethyl group and attached to the aromatic ring that represents a strong electrostatic field. Figure 2 portrays the hydrogen-bond and hydrophobic interaction patterns. This binding conformation of the COX-2 receptor in the binding site is supposed to a reason for their significant inhibitory effect against COX-2 activity. Hence, we hypothesized of the selected top compound ZINC000039428234can be tested for breast cancer for their biological activity through in vivo/in vitro studies for the development of breast drug candidates against COX-2 protein.

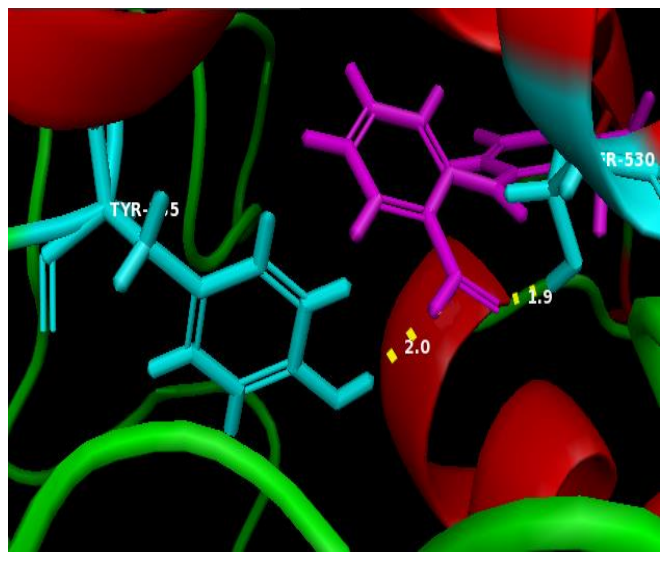

A

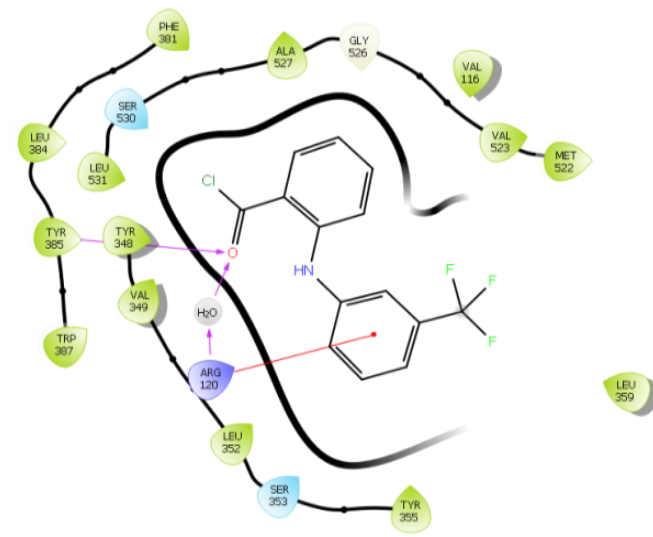

B

Figure 2: (A) Hydrogen bonds interaction between the receptor and ligand (ZINC000039428234). (B) Hydrogen bonding between the compound and COX-2 enzyme (PDB ID: 5IKR) amino acids

Molecular Dynamic simulations provide an accurate ranking of the potential ligands, and in the latest developments, during the docking process itself to find the binding site, time dynamics of interacting amino acid residues and correctly dock the ligand a priori[33]. Three different software for MD simulations were used in this study that has been mentioned previously and represented in Figure 3. By computationally exhaustive exploratory molecular dynamics simulation using CABS-Flex 2.0 [35], we obtained 10 different models. We select the first model because of its best structural heterogeneity, their optimum free energy, and highly stable configuration.

We ran MD simulations for the COX-2 protein complex and run in for $10 \mathrm{~ns}$. Initially, the RMSF plot showed that the complex deviated for a certain period and attained equilibrium at $10 \mathrm{~ns}$. Subsequently, it remained stable throughout the simulation time for up to $30 \mathrm{~ns}$. As per our findings, the complex has many fluctuations in chain A, the highest amplitude being of residue 158 of around $5.4 \AA$. The rest of the fluctuations found in the complex structure during MD simulation have been presented in Table 3. The RMSF plot has also been depicted in Figure 4 using Cabs-Flex 2.0. 
MD simulation by CABS- Flex 2.0 [35] provides the best stable configuration of the protein complex. After the MD simulation, we discern that our protein structure has helices and many loops, which could refer to the fact that our protein has some disordered structures or some missing residues because of which we also obtained many fluctuations in the same. It also indicates that our protein might have undergone some post-translational modifications (PTMs) during the simulation. In order to determine those modifications, we executed Vienna-PTM MD simulations which provide evidence to alterations in the protein structure during MD simulation analysis [37]. To our amazement, we observed that MD simulation using Vienna-PTM gives a better protein structure when compared to CABS-Flex 2.0 as the loops are drastically reduced and helices increased with a heavier structure. The advantage of Vienna-PTM over CABS-Flex 2.0 is the fact that it uses GROMACS MD forcefield parameters for simulation (GROMOS-45A, 54A7, 54A8) and uses modified amino acids to enhance the sampling. We also utilized a Poisson-Boltzmann/Generalized Born (PB/GB) molecular mechanics, viz., Bluues [36] in order to compute the electrostatics of our protein of interest. We observed that there were a total of 1102 residues and 17651atoms present in our input structure. The computed electrostatics computed was observed as - Born self-energy: $0.0(\mathrm{~kJ})$, Coulomb energy: 0.0 $(\mathrm{kJ})$, Electrostatic solvation energy: $0.0(\mathrm{~kJ} / \mathrm{mol})$. The total energy was calculated as 5724.165585 $(\mathrm{kJ} / \mathrm{mol})$.

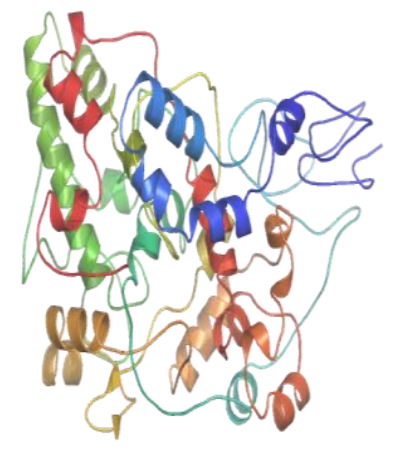

A

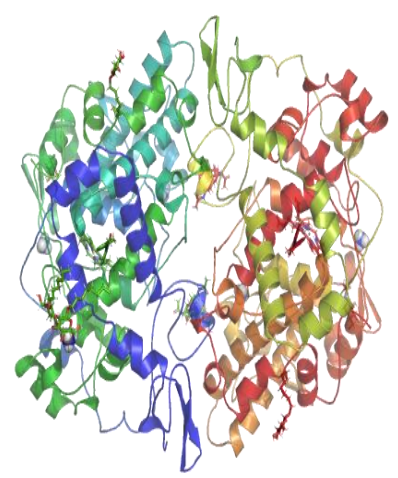

B

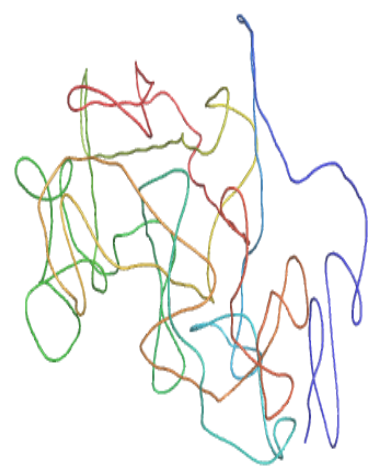

C

Figure 3: Stable protein structure after MD simulations (a) using CABS-Flex 2.0 and (b) using Vienna-PTM and (c) using Bluues

\begin{tabular}{|l|l|}
\hline Residue Index (Chain A) & RMSF Value $(\AA)$ \\
\hline 158 & 5.4 \\
\hline 230 & 3.8 \\
\hline 35 & 4.5 \\
\hline 358 & 3.2 \\
\hline 383 & 3.2 \\
\hline 508 & 3.5 \\
\hline
\end{tabular}

Table 3. Root Mean Square Fluctuations (RMSF) present in the protein complex. 


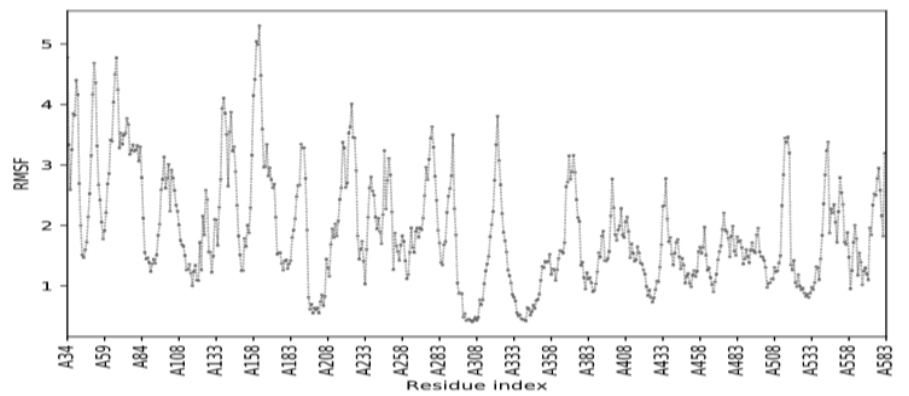

Figure 4: Root Mean Square Fluctuation (RMSF) plot of the protein complex.

The structured generated using Bluues was highly unstable as it produced enormously high free energy and the simulated structure lacked helices and strands but were simply loopy in nature. Titrable residues, GB radii, pKA residues, residues accessible to the solvent are provided as supplementary. Figure 5 gives a comparative reflection of the three different MD simulations, the first one by CABS-Flex 2.0 and second by Vienna-PTM and third by Bluues respectively. Proteincompound ZINC000039428234 complex was analyzed to extend the information obtained from previous docking studies. The flexibility of the enzyme backbone structure and its stability has been shown in figure 6. This complex structure attained a high level of fluctuation in the residue positions 160 was discerned to be $3.8 \AA$.

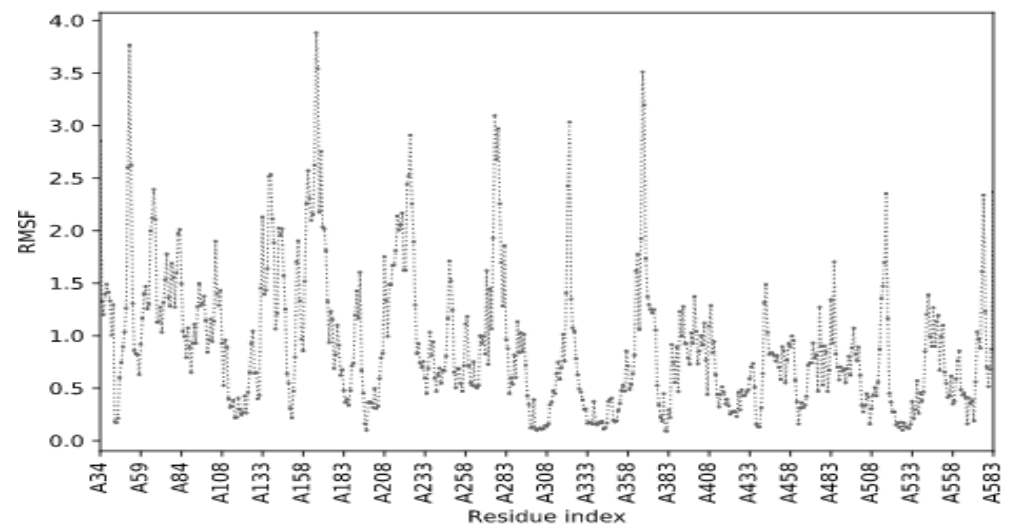

Figure 5: Root Mean Square Fluctuations plots of protein structure with compound ZINC000039428234

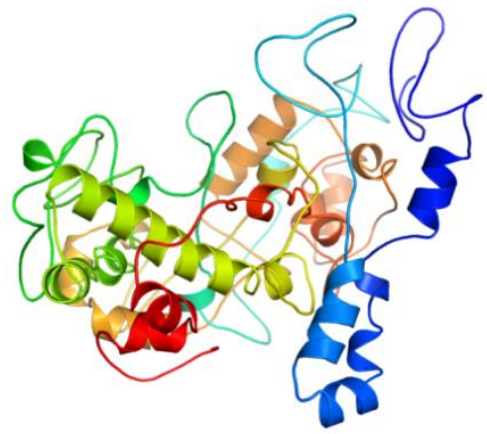

Figure 6: Stable structures generated after MD Simulation of compound ZINC000039428234 


\section{Conclusion}

The present study retrieves COX-2 inhibitors based on molecular docking, ADMET as well as MD simulation properties. This research work deals with the selected COX-2 enzyme using in silico approach. The screening process successfully identifies 11 best compounds against COX-2 protein that have high binding affinity from different databases compared to the native compound. All the identified compounds tested for the rule of five using the Qikprepprogram and Molinspiration server for determining the drug-likeness properties and they were following the Lipinski rule of five. We reported that the compound ZINC000039428234 $(-10.738 \mathrm{kcal} / \mathrm{mol})$ was a good inhibitor against COX-2 protein and further used for the receptor-ligand interaction and MD simulation analysis. The selected compound, having illustrated the most favorable result so may represent another essential contribution to the development of drug candidates against COX-2 protein to the arsenal of breast cancer therapy. We outlined the identification of COX-2 inhibitor that may be subjected to further wet laboratory confirmations for possible use as alternatives for the treatment of breast cancer. These best hits will not only provide the lead compounds but also suggest that the favorable find a new path to designing novel COX inhibitors. By executing MD simulation using three different approaches, we can discern that we must deploy the best stable and efficient structure which has optimal free energy for further analysis.

\section{Acknowledgments}

AS acknowledges funding from the Indian Council of Medical Research, New Delhi (Grant No. 3/1/3/PDF(14)2016-HRD) for financial assistance. SQ is supported by DST-Inspire Fellowship, Department of Science \& Technology, Government of India.

\section{References}

[1] Chandrasekharan NV, Simmons DL. The cyclooxygenases. Genome Biol 2004;5:241.

[2] Clària J. Cyclooxygenase-2 biology. Curr Pharm Des 2003;9:2177-90.

[3] Chow LWC, Loo WTY, Toi M. Current directions for COX-2 inhibition in breast cancer. Biomed Pharmacother 2005;59 Suppl 2:S281-284.

[4] Botting RM. Inhibitors of cyclooxygenases: mechanisms, selectivity and uses. J Physiol Pharmacol 2006;57 Suppl 5:113-24.

[5] Ghosh N, Chaki R, Mandal V, et al. COX-2 as a target for cancer chemotherapy. Pharmacol Rep 2010;62:233-44.

[6] Bajorath J. Computer-aided drug discovery. F1000Res 2015;4.

[7] Meng X-Y, Zhang H-X, Mezei M, et al. Molecular Docking: A powerful approach for structurebased drug discovery. Curr Comput Aided Drug Des 2011;7:146-57.

[8] Ferreira LG, Dos Santos RN, Oliva G, et al. Molecular Docking and Structure-Based Drug Design Strategies. Molecules 2015;20:13384-421.

[9] Chang S-S, Huang H-J, Chen CY-C. High performance screening, structural and molecular dynamics analysis to identify H1 inhibitors from TCM Database@Taiwan. Mol Biosyst 2011;7:3366-74.

[10] Kitchen DB, Decornez H, Furr JR, et al. Docking and scoring in virtual screening for drug discovery: methods and applications. Nat Rev Drug Discov 2004;3:935-49.

[11] Cheng F, Li W, Liu G, et al. In silico ADMET prediction: recent advances, current challenges and future trends. Curr Top Med Chem 2013;13:1273-89. 
[12] Sirois J, Sayasith K, Brown KA, et al. Cyclooxygenase-2 and its role in ovulation: a 2004 account. Hum Reprod Update 2004;10:373-85. 13 Berman HM, Westbrook J, Feng Z, et al. The Protein Data Bank. Nucleic Acids Res 2000;28:235-42.

[14] Sastry GM, Adzhigirey M, Day T, et al. Protein and ligand preparation: parameters, protocols, and influence on virtual screening enrichments. J Comput Aided Mol Des 2013;27:221-34.

[15] Hollingsworth SA, Karplus PA. A fresh look at the Ramachandran plot and the occurrence of standard structures in proteins. Biomol Concepts 2010;1:271-83.

[16] Laskowski RA, MacArthur MW, Moss DS, et al. PROCHECK: a program to check the stereochemical quality of protein structures. J Appl Cryst 1993;26:283-91.

[17] Zhou AQ, O'Hern CS, Regan L. Revisiting the Ramachandran plot from a new angle. Protein Sci 2011;20:1166-71.

[18] Elokely KM, Doerksen RJ. Docking challenge: Protein sampling and molecular docking performance. J Chem Inf Model 2013;53:1934-45.

[19] Friesner RA, Banks JL, Murphy RB, et al. Glide: a new approach for rapid, accurate docking and scoring. 1. Method and assessment of docking accuracy. J Med Chem 2004;47:1739-49.

[20] Friesner RA, Murphy RB, Repasky MP, et al. Extra precision glide: docking and scoring incorporating a model of hydrophobic enclosure for protein-ligand complexes. J Med Chem 2006;49:6177-96.

[21] Pence HE, Williams A. ChemSpider: An Online Chemical Information Resource. J Chem Educ 2010;87:1123-4.

[22] Ayers M. ChemSpider: The Free Chemical Database. Reference Reviews Published Online First: 14 September 2012.

[23] Gilson MK, Liu T, Baitaluk M, et al. BindingDB in 2015: A public database for medicinal chemistry, computational chemistry and systems pharmacology. Nucleic Acids Res 2016;44:D1045-1053.

[24] Kristensen TG, Nielsen J, Pedersen CNS. METHODS FOR SIMILARITY-BASED VIRTUAL SCREENING. Computational and Structural Biotechnology Journal 2013;5:e201302009.

[25] Greenwood JR, Calkins D, Sullivan AP, et al. Towards the comprehensive, rapid, and accurate prediction of the favorable tautomeric states of drug-like molecules in aqueous solution. $J$ Comput Aided Mol Des 2010;24:591-604.

[26] Shelley JC, Cholleti A, Frye LL, et al. Epik: a software program for pKaprediction and protonation state generation for drug-like molecules. J Comput Aided Mol Des 2007;21:681-91.

[27] Genheden S, Ryde U. The MM/PBSA and MM/GBSA methods to estimate ligand-binding affinities. Expert Opin Drug Discov 2015;10:449-61.

[28] Norinder U, Bergström CAS. Prediction of ADMET Properties. ChemMedChem 2006;1:920-37.

[29] Lipinski CA. Rule of five in 2015 and beyond: Target and ligand structural limitations, ligand chemistry structure and drug discovery project decisions. Adv Drug Deliv Rev 2016;101:34-41.

[30] Lipinski CA. Drug-like properties and the causes of poor solubility and poor permeability. $J$ Pharmacol Toxicol Methods 2000;44:235-49.

[31] Sterling T, Irwin JJ. ZINC 15 - Ligand Discovery for Everyone. J Chem Inf Model 2015;55:2324-37.

[32] Lipinski CA. Lead- and drug-like compounds: the rule-of-five revolution. Drug Discov Today Technol 2004;1:337-41.

[33] Karplus M, McCammon JA. Molecular dynamics simulations of biomolecules. Nat Struct Biol 2002;9:646-52.

[34] Alonso H, Bliznyuk AA, Gready JE. Combining docking and molecular dynamic simulations in drug design. Med Res Rev 2006;26:531-68. 
[35] Kuriata A, Gierut AM, et al. CABS-flex 2.0: a web server for fast simulations of flexibility of protein structures. Nucleic Acids Research 2018; 46(W1): W338-W343.

[36] Fogolari F, Corazza A, et al. Bluues: a program for the analysis of the electrostatic properties of proteins based on generalized Born radii.BMC Bioinformatics2012;13, S4-S18.

[37] Margreitter C, Petrov D \& Zagrovic B. Vienna-PTM web server: a toolkit for MD simulations of protein post-translational modifications. Nucleic Acids Research 2013; 41(Web Server issue):W422-6. 